\title{
Post-translational modifications of FOXO family proteins (Review)
}

\author{
ZIYAO WANG ${ }^{1}$, TINGHE YU ${ }^{2}$ and PING HUANG ${ }^{1}$ \\ ${ }^{1}$ National Key Clinical Department, Department of Hepatobiliary Surgery, \\ The First Affiliated Hospital of Chongqing Medical University; ${ }^{2}$ Chongqing Key Medical Laboratory of Obstetrics and Gynecology, \\ The Second Affiliated Hospital of Chongqing Medical University, Chongqing Medical University, \\ Chongqing 400000, P.R. China
}

Received September 23, 2015; Accepted September 21, 2016

DOI: $10.3892 / \mathrm{mmr} .2016 .5867$

\begin{abstract}
The Forkhead box O (FOXO) protein family is predominantly involved in apoptosis, oxidative stress, DNA damage/repair, tumor angiogenesis, glycometabolism, regulating life span and other important biological processes. Its activity is affected by a variety of posttranslational modifications (PTMs), including phosphorylation, acetylation, ubiquitination, methylation and glycosylation. When cells are subjected to different environments, the corresponding PTMs act on the FOXO protein family, to change transcriptional activity or subcellular localization, and the expression of downstream target genes, will ultimately affect the biological behavior of the cells. In this review, we will discuss the biological characteristics of FOXO protein PTMs.
\end{abstract}

\section{Contents}

1. Introduction

2. Phosphorylation of FOXO proteins

3. Acetylation of FOXO proteins

4. Ubiquitination of FOXO proteins

5. Methylation of FOXO proteins

6. Glycosylation of FOXO proteins

7. Conclusion

Correspondence to: Professor Ping Huang, National Key Clinical Department, Department of Hepatobiliary Surgery, The First Affiliated Hospital of Chongqing Medical University, Chongqing Medical University, 1 Youyi Road, Yuzhong, Chongqing 400000, P.R. China

E-mail: huangpchina@sina.com

Key words: FOXO proteins, posttranslational modifications, activity, sites

\section{Introduction}

In 1989, Weigel et al (1) first cloned the fork genes (fkh) from fruit flies, and discovered that the encoded protein was essential for the normal development of embryos $(1,2)$. Currently, researchers have identified $>100$ types of Forkhead box (Fox) proteins present in almost all eukaryotes from yeast to humans (3). Fox protein families have a conserved DNA-binding region, which can specifically bind to the conserved DNA sequence 5'-TTGTTTAC-3' (4). The spatial structure of the Fox protein exhibits a 'helix-turn-helix' structure, which resembles a fork, thus, providing the name 'fork proteins' (5). Furthermore, through analyzing the similarity of the conserved region of the amino acid sequence, Fox proteins are divided into the 'A' and ' $S$ ' subtypes $(6,7)$. At present, the FOXO protein family is one of the most widely studied protein families. In mammalian species, the FOXO subfamily includes FOXO1, FOXO3, FOXO4 and FOXO6. FOXO1, FOXO3 and FOXO4 are widely expressed in various tissues and organs (8), however the expression of FOXO6 is most often detected in the developing brain (9), and recent studies have demonstrated that FOXO6 is involved in cell growth and transformation of liver cancer and lung cancer $(10,11)$. FOXO homolog genes, DAF-16 and dFOXO, exists in lower organisms, including Caenorhabditis elegans, Drosophila and sponges (12).

FOXO proteins control a series of cellular behaviors, including apoptosis, cell cycle, cell differentiation, cell proliferation, energy metabolism and autophagy (13), by activating or inhibiting downstream targets, via transcriptional regulatory functions. As FOXO proteins have important effects on the cell, changes in the expression of FOXO proteins are associated with the physiological or pathological processes of aging, angiogenesis, cancer, diabetes, infertility (14), immune system disorders (15) and neurodegeneration (16).

FOXO proteins are activated by various extracellular stimuli, including growth factors, cytokines and hormones. In order to further reveal the molecular mechanisms of FOXO proteins in transcriptional regulation, more studies have focused their attention on posttranslational modifications (PTMs). Currently, there are $>400$ types of protein PTMs have been determined, including phosphorylation, acetylation, 
ubiquitination, methylation, glycosylation, small ubiquitin-like modification and nitrosylation. Different PTMs change the expression of FOXO target genes, and affect the activity, subcellular localization, DNA binding activity and half-life of FOXO proteins via different enzymatic reactions (17).

This review aims to summarize the PTM mechanisms that regulate FOXO proteins, and to further clarify the transcriptional regulatory role of FOXO proteins and detect novel drug targets for cancer therapy.

\section{Phosphorylation of FOXO proteins}

Phosphorylation, induced by a variety of protein kinases, is the most important PTM of FOXO proteins. Different enzymes regulate phosphorylation at different sites on FOXO proteins to produce different biological effects (Table I).

Akt serine threonine kinase (Akt) and serum/glucocorticoid regulated kinase ( $S G K)$. Akt (also termed protein kinase B) and SGK are major downstream targets of the phosphatidylinositol 4,5-bisphosphate 3-kinase (PI3K) pathway, and furthermore, are the most extensively researched serine/threonine kinases involved in FOXO protein phosphorylation. These two kinases recognize the same phosphorylation substrate motif, RXRXXS/T (R, arginine; $\mathrm{X}$, any amino acid; and $\mathrm{S} / \mathrm{T}$, serine/threonine). Akt phosphorylates FOXO1 at three sites, in particular, phosphorylation at Ser256 can change the positive charge of FOXO1 DNA binding domain to negative, thus, reducing the activity of FOXO1 $(18,19)$. Akt and SGK phosphorylate FOXO3 at same sites, however SGK preferentially phosphorylates at Ser315, and Akt at Ser253. It has been reported that the process of FOXO3 phosphorylation is regulated by Akt and SGK synergistically (20).

In addition, the binding of FOXO with 14-3-3, which is a key protein involved in the translocation of FOXO proteins from the nucleus to the cytoplasm, requires FOXO phosphorylation at specific sites. For example, following phosphorylation, FOXO3 can combine with 14-3-3, this significantly changes the structure of the FOXO nucleus localization signal. Typically, binding to 14-3-3 increases nuclear export of FOXO proteins and decreases reentry into the nucleus $(21,22)$. Additionally, the dissociation of FOXO3 from 14-3-3 is mediated by protein phosphatase 2 , which dephosphorylates FOXO3 at Thr32/Ser253 (22). Studies have reported that phosphorylation of FOXO1 at Thr24 by Akt is necessary and sufficient for its binding to 14-3-3, however, when exclusion of FOXO1 from the nucleus id induced by insulin-like growth factor-1 (IGF-1), combining with 14-3-3 is not required. This result suggests that the translocation process of FOXO proteins is not only 14-3-3-dependent (23).

Changing the subcellular distribution of the FOXO proteins is not the only way to reduce its transcriptional activity. For example, FOXO1 transcriptional activity can be inhibited by activation of Akt signaling pathways induced by insulin, however, when the key site (Leu375) of the nuclear export signal, which regulates the transfer of FOXO1 to the cytoplasm, is mutated to alanine, FOXO1 loses the ability to relocalize, however, notably, insulin, remains able to inhibit FOXO protein activity (24). Furthermore, FOXO4 phosphorylation at Thr28 and Ser193 downregulates the transcription activity of the protein by suppressing DNA binding $(22,25)$. In addition, Akt also induces the degradation of FOXO via the proteasomal pathway (26-28). The E3 ligase, S-phase kinase associated protein 2 ( $\mathrm{Skp} 2$ ), suppresses FOXO1 transactivation, and eliminates its effect on inhibition of cell proliferation. The normal function of Skp2 phosphorylates the Ser256 site of FOXO1, and subsequently, causes degradation of FOXO1 via the proteasomal pathway (29).

Akt achieves its function of phosphorylating to regulating FOXO proteins though the interaction with various factors that form a complex loop (30). PKR-like ER kinase (PERK) phosphorylates FOXO1 at Ser298, Ser301 and Ser303, but prefers Ser298 site, which is not a target of Akt. Notably, PERK phosphorylates FOXO1 and increased the transcriptional activity, which is in contrast with the effect of Akt. This suggests that FOXO phosphorylation induced by PERK can counteract the phosphorylation effect of Akt. Research has demonstrated that PERK can directly upregulate the activity of FOXO proteins, or decrease its activity via indirect effects on Akt. Furthermore, the effects of PERK on FOXO protein increases the expression of insulin receptor, which increases the activity of AKT, and the activation of AKT, in turn, inhibits the activity of the PERK (31). Thus, the higher activation of AKT, the less PERK activity, comparably $(32,33)$.

Mammalian sterile 20-like kinase (MST) and Jun- $N$-terminal kinase (JNK). MST has an important role in the regulation of cell size and apoptosis. Upon oxidative stress, MST1 binds and phosphorylates FOXO3 $(34,35)$. Phosphorylation of FOXO3 mediated by MST1 disrupts its connection with 14-3-3, prompting the accumulation of it in the nucleus, and therefore, upregulates the expression of pro-apoptotic genes that induce neuronal cell death (36). Certain studies have reported that MST1 can affect the expression of downstream target genes of FOXO1 by similar mechanisms (37). Furthermore, the MST1-FOXO pathway has a significant role in drug treatment-induced cancer cell death (38). When cells are under to oxidative stress, the JNK-dependent signaling pathway causes phosphorylation of $\mathrm{FOXO} 4$, thereby inducing FOXO4 nuclear translocation and activity (39). JNK mediates the FOXO activity via the correct assembly of JNK interacting protein 1 (JIP1) complexes, which includes three substrates, mitogen-activated protein kinase (MAPK) kinase kinase 11, MAPK kinase 4 and JNK. The correct assembling of this complex is involved in the function of the JNK signaling pathway, thus, it can affect the activity of FOXO transcription factors directly. Additionally, Ras-like proto-oncogene A (RALA) is a small GTPases of the Ras superfamily and induces the correct assembly of the JIP1 scaffolding protein complex. Via reactive oxygen species (ROS) and RALA-dependent pathways, FOXO4 is induced and fully phosphorylated $(39,40)$. The activation of FOXO induced by JNK, not only depends on its phosphorylation, but also on other interacting proteins or pathways. For example, JNK phosphorylates 14-3-3 and releases FOXO3 from 14-3-3, which antagonizes the effects of Akt signaling (41). In pancreatic HIT cells, the JNK signaling pathway may reduce the activity of Akt and, thus, reduce the phosphorylation of FOXO1 (42). It has been reported that JIP1 serves a key role 
in the regulation of Akt activity, however the specific mechanism remains unclear (43).

Extracellular signal-regulated kinase $($ ERK)/p38. MAPKs include three members, ERK, JNK and p38. Under oxidative stress, FOXO1 is phosphorylated by ERK and p38 cooperatively (44). Accordingly, p38 can induce phosphorylation of FOXO3 following treatment with doxorubicin (45). Recent studies demonstrated that FOXO3 phosphorylation mediated by $38 \beta$, upregulates BCL2 interacting protein 3 expression, resulting in mechanistic target of rapamycin complex 1 inhibition and cell apoptosis (46). ERK1/2 can directly phosphorylate FOXO3a, and phosphorylated FOXO3a is subsequently degraded by MDM2 proto-oncogene (MDM2), which is similar to E3 ligase Skp2, to negatively regulate the transcriptional activity of FOXO3a (47). In addition, ERK can decrease the activity of the FOXO protein through phosphorylation of other proteins. Previous studies have reported the association between ERK, p66 shc adaptor protein 1 (p66shcA) and FOXO3. The phosphorylation of p66shcA Ser36 is required for phosphorylation of FOXO3a, which can be induced by ERK1/2 (48). In cardiac fibroblasts, activation of ERK1/2 can directly phosphorylate FOXO3a and regulate its activity, and ERK1/2 can also phosphorylate Skp2 in the same manner. Notable, a report previously demonstrated that Skp2 can inhibit the activity of FOXO3a and promote its degradation $(48,49)$. SGK is another link between MAPK and FOXO proteins. When DNA is damaged, phosphorylated SGK1 reduces the activity of FOXO3a through ERK1/2. In addition, ERK and Akt can regulate the function of each other, for example, following oxidative stress, inhibiting PI3K can change the distribution of ERK1/2 phosphorylation in the cell. However, inhibition of ERK can also affect the phosphorylation of AKT (50).

Cyclin-dependent kinases (CDKs). The initiation of the cell cycle depends on the activity of complexes of cyclins and CDKs. CDK2 can specifically phosphorylate FOXO1 at Ser249, and induces transfer of FOXO1 to the cytoplasm, ultimately reducing its transcriptional activity (51). In addition to CDK2, CDK1 can also phosphorylate FOXO1 at Ser249 (52). Research has demonstrated that, in prostate cancer cells, CDK1 phosphorylates FOXO1 at Ser249, thereby, inhibiting the transcriptional activity of FOXO1 and reducing the effects of FOXO1 on mitosis. FOXO1 phosphorylation induced by CDK1 not only eliminates the effect of FOXO1 on cell death, but also reduces the inhibitory effects of FOXO1 on the proliferation of malignant transformed cells (53). In addition to CDK1 and CDK2, a recent study suggested that the CDK5 can influence the transcriptional activity of FOXO1 directly. Different extracellular stimuli, including oxidative stress, ischemia reperfusion and serum starvation, can induce CDK5, which leads to phosphorylation of FOXO1 at Ser249. Furthermore, the CDK5/P25 complex significantly increases the binding of FOXO1 and 14-3-3 protein, thereby promoting FOXO1 retention in the cytoplasm, and ultimately, inhibits its transcriptional activity. Phosphorylation of FOXO1 at Ser249 mediated by CDK5 can inhibit the retention of FOXO1 in the cytoplasm induced by downregulation of Akt activity. This research suggests that CDK5 may regulate the function of FOXO1 by direct and indirect mechanisms (54).
Adenosine monophosphate-activated protein kinase (AMPK). In mammals, AMPK phosphorylates human FOXO3 at six regulatory sites (Table I) (55). The inactivation of p38 $\alpha$ triggers nuclear translocation of FOXO3a in an AMPK-dependent manner, and leads to subsequent activation of FOXO3a targets genes, which induces autophagy, cell-cycle arrest and cell death (56). AMPK activity is necessary for defending against ROS-induced injury, and the molecular mechanism responsible for this effect. AMPK directly phosphorylates human FOXO1 at Thr649, which is critical for FOXO1 nuclear localization, stabilization and transcriptional activity, and the phosphorylation event leads to increased expression of the anti-oxidant enzymes, manganese superoxide dismutase (MnSOD) and catalase (57).

AMPK-FOXO3a signaling is critical for regulation of energy homeostasis and oxidative stress in cells (58). It has been reported that the activation of AMPK by 5-aminoimidazole-4-carboxamide ribonucleotide (AICAR) results in increased FOXO1 mRNA and protein levels, and strongly enhances FOXO1 transcriptional activity (59). FOXO3a is also induced via AMPK, and this effect promotes its nuclear and mitochondrial translocation, resulting in significantly reduced ROS levels, enhanced aerobic respiration and maintaining energy homeostasis (60). Furthermore, prolonged activation of AMPK by AICAR enhances the nuclear import of FOXO3a and improves the adaptability of cells to oxidative stress (61). During hypoxia, AMPK is important for in regulating FOXO proteins in cells. For example, in anoxic myocardial cells, upregulation of AMPK can decrease the phosphorylation level of FOXO1 and FOXO3, and exclusion of them from the nucleus, leading to sequential activation of target genes, such as catalase (62). It has been demonstrated that AMPK phosphorylates the FOXO homologue, DAF-16, at multiple sites and activates DAF-16-dependent transcription (63). Notably, DAF-16 can induce the expression of genes encoded by AMPK, which slows aging in Caenorhabiditis elegans. This suggests that DAF-16 and AMPK form a positive feedback loop (64). In mammals, however, the research about this feedback loop has not detected any changes in the $\gamma$ substrate of AMPK depending on FOXO protein activation (65). However, it cannot be ruled out that FOXO proteins tissue-specifically modulate the gene transcription of AMPK, as human FOXO3 and FOXO4 directly induce liver kinase B1, which phosphorylates AMPK and enhances its bioactivity (66). Thus, it is hypothesized that a FOXO-AMPK pathway feedback loop may exist in mammalian cells.

In addition to directly phosphorylating and forming a feedback loop with FOXO proteins, AMPK can also modulate other proteins in order to regulate FOXO proteins. For example, AMPK, by increasing the levels of $\mathrm{NAD}^{+}$, increases the activity of histone deacetylation enzyme, sirtuin 1 (SIRT1), which consequently leads to the activation and acetylation of FOXO1 and FOXO3 (67).

$I \kappa B$ kinase $(I \kappa K)$. I $\mathrm{K} \mathrm{K}$, a central regulator of nuclear factor- $\kappa \mathrm{B}(\mathrm{NF}-\kappa \mathrm{B})$, is involved in controlling cell proliferation, survival, the prevention of apoptosis and tumorigenesis.

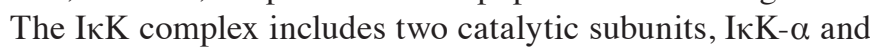

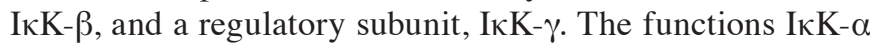




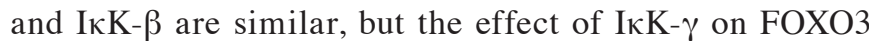
remains unclear. FOXO3 phosphorylation at Ser644 induced by IкK- $\beta$ promotes its retention in the cytoplasm, and thus, has a pivotal role in chemotherapeutic resistance. Furthermore, the overexpression of I $\kappa \mathrm{K}-\beta$ results in an accumulation of endogenous $\mathrm{FOXO} 3$ in the cytoplasm and degradation via the ubiquitin proteasome pathway. Also, using anti-NEMO binding domain peptide significantly inhibits the IкK complex, which induces the relocalization of FOXO3 to the nucleus. Overall, the phosphorylation of FOXO by IкK decreases its stability, and also changes its subcellular loca-

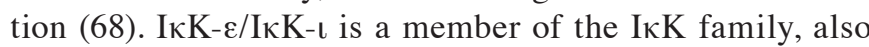
known as IKBKE. Previous studies have demonstrated that inflammatory stimuli, including interferon (IFN) and viruses, activate I $\kappa \mathrm{K}-\alpha / \beta$ and IKBKE. The IKBKE kinase

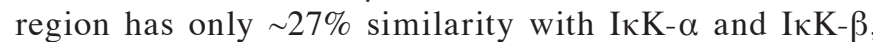
but it can phosphorylate FOXO3a at Ser644, leading to the degradation of FOXO3a and export from the nucleus, thus reducing the transcriptional activity of FOXO3a (69). Additionally, phosphorylation of FOXO3a by IKBKE does not rely on the Akt pathway (70). Recent research suggests that IKBKE, which activates Akt, can directly phosphorylate Akt at Thr308 and Ser473, therefore, IKBKE regulates FOXO3a activity directly, and also regulates the activity of FOXO3 indirectly via the Akt pathway (71). In addition, it has been demonstrated that phosphorylation of FOXO3a caused by IKBKE decreases the expression of IFN- $\beta$ to participate in the regulation of the immune response (72). In acute myeloid leukemia cells, FOXO3 is in a constitutively inactive state due to its cytoplasmic localization, which is not dependent on the $\mathrm{PI} 3 \mathrm{~K} / \mathrm{Akt}$ or ERK/MAPK pathways, instead, NF- $\mathrm{KB}$, which is a key regulator of cell survival, sustains a constitutively active state (73). IкK-specific inhibition upregulates FOXO3 and tumor necrosis factor (TNF)-related apoptosis-inducing ligand (TRAIL) (74). It has also been reported that FOXO3a

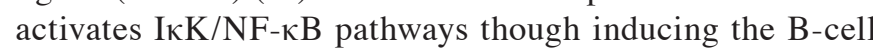
CLL/lymphoma 10 protein (BCL10), which is an upstream

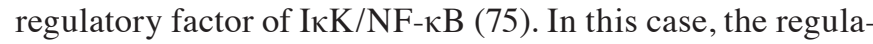
tion of FOXO3 and I $\kappa \mathrm{K}$ is reciprocal.

\section{Acetylation of FOXO proteins}

Similarly to phosphorylation, acetylation also regulates various functions of different FOXO proteins. Deacetylases and acetylases have been reported to modify FOXO proteins to change their DNA-binding activity, stability and interaction with other proteins.

Deacetylation. The effect of acetylation on FOXO proteins is controlled by the histone acetyltransferases and histone deacetylases (HDACs). HDACs, which remove the acetyl groups from histones though enzymatic reaction, are pivotal for regulating gene expression. HDACs include class I (HDAC1, 2, 3 and 8), class II a (HDAC4, 5, 7 and 9), class II b (HDAC6 and 10), class III and class IV (HDAC11). The class III HDACs, also termed sirtuins, possess NAD-dependent catalytic sites and traditional inhibitors of HDACs cannot inhibit sirtuin function (76). Mammals have seven sirtuins, with sirtuin (SIRT) 1-3 exhibiting strong NAD ${ }^{+}$-dependent deacetylation activity, whereas SIRT4-7 exhibit strong deacetylation and are more accurately described as $\mathrm{NAD}^{+}$-dependent deacylases (77). SIRT2 is predominantly localized in the cytoplasm, whereas SIRT3, SIRT4 and SIRT5 are predominantly in the mitochondria, and SIRT1, SIRT6, SIRT7 in the nucleus (78). FOXO proteins are deacetylated by HDACs and sirtuins simultaneously. Certain studies indicate that class I and II HDACs regulate FOXO nuclear localization and transcriptional activation in response to nutrient deprivation (79). However, compared with class II, class I HDACs preferentially regulate the activity of FOXO proteins. Generally, HDAC1 is able to complex with HDAC2 to exert normal functions. In addition, HDAC4/5 recruits HDAC 3 and FOXO proteins to form a complex, allowing it to be deacetylated and activated (80). It has been reported that $\mathrm{HDAC} 1$ is a primary regulator of FOXO in skeletal muscle and a key regulator of the atrophy. Furthermore, HDAC1-mediated deacetylation of FOXO in the cytosol may be a major signal that leads to decreased phosphorylation and nuclear localization of FOXO (79). Research has demonstrated that reducing FOXO3a acetylation may lead to FOXO3a-dependent transcriptional activation and induce atrophy of muscle fibers $(81,82)$. There is substantial literature demonstrating that SIRT1 can deacetylate FOXO1, FOXO3a and FOXO4, and subsequently, stimulate the expression of anti-oxidants, including MnSOD and thioredoxin (83), and through an auto-feedback loop also potentiate SIRT1 expression (84). A previous study demonstrated that SIRT1 has a primary role in the regulation of endothelial progenitor cell apoptosis induced by oxidative stress, and that SIRT1 integrated with FOXO3a directly to inhibit FOXO3a by deacetylation (85). SIRT1 and SIRT2, through deacetylating FOXO3, promote the degradation of FOXO3 via the ubiquitin-proteasome pathway. Studies have demonstrated that Skp2, which is the substrate of E3 ubiquitin ligase, prefers to combine with acetylated FOXO3, where Lys242, Lys259, Lys290 and Lys569 sites of acetylated FOXO3 are targets for ubiquitination (86), thus, acetylation of FOXO3a exposes the lysine groups, and thus, promotes FOXO3a polyubiquitination.

SIRT1 mediated multitudinous biological effects though deacetylation of FOXO proteins. It was reported that SIRT1 increases the activity of FOXO proteins by deacetylation, however, others have demonstrated that the functions of FOXO proteins are negatively correlated with deacetylation induced by SIRT1 (87). Indeed, deacetylation of FOXO proteins increases the transcription activity (88). Kitamura et al (88) reported that compared with wild-type FOXO1, mutated FOXO1, that cannot be acetylated, has stronger transcriptional activity. However, it has been recently demonstrated that acetylated FOXO proteins are easily degraded by the ubiquitin-proteasome pathway, which abolishes the increased transcriptional activity of FOXO proteins induced by deacetylation (86). To summarize, this mechanism may be a crucial step for balancing the effect of FOXO on transcription. Typically, SIRT1 is located in the nucleus, however, SIRT1 has also been reported to be detected in the cytoplasm or mitochondria (89). In fact, SIRT1, when located in the cytoplasm, promotes autophagy and nuclear localization (90). However, SIRT2 is predominantly localized in the cytoplasm. In the nucleus, SIRT1 induces autophagy though deacetylation of FOXO1 and activates FOXO1 transcriptional activity. When 
SIRT1 is deactivated, acetylated FOXO1 is exported to the cytoplasm, and combines with the autophagy related 7 protein to accelerate autophagy. Whereas, SIRT2-induced deacetylation of FOXO1 inhibits autophagy (91). Therefore, SIRT1 and SIRT2 exhibit opposing effects on the regulation of certain cell behaviors, even though they both deacetylate FOXO1. Resveratrol can increase the SIRT1 deacetylation activity, which reduces FOXO1 and FOXO3a acetylation and increases the nucleus localization, resulting stronger transcriptional activity. Resveratrol activates AMPK, which increases the deacetylase activity of SIRT1, and further increases the FOXO1 transcription activity $(92,93)$. Studies have demonstrated that certain proteins form complexes with FOXO-SIRT1. The protein four and a half LIM domains 2 (FHL2) improves the interaction between SIRT1 and FOXO1. In prostate cancer cells, FOXO1 activity is inhibited by FHL2, which promotes the deacetylation of FOXO1 by SIRT1 (94). FoxO1 protects pancreatic beta cells against oxidative stress by forming a complex with the promyelocytic leukemia protein and the NAD-dependent deacetylase SIRT1, resulting in activation of neurogenic differentiation 1 and MAF bZIP transcription factor $\mathrm{A}$, which are two Insulin 2 gene transcription factors (90). In addition, lysine demethylase 5B (KDM5) protein can also form a complex with HDAC4 and combine with FOXO proteins, thereby promoting FOXO deacetylation. The complex binds to target genes with FOXO promoter regions to promote resistance to expression of oxidative stress proteins. In the absence of KDM5, HDAC4 can still form a complex with FOXO1 and induce its deacetylation; however, FOXO1 recruitment to a subset of target genes, which have a role in oxidative stress resistance, is attenuated. This decreased ability of FOXO1 induces the decreased transcriptional activation of a subset of oxidative stress resistance genes (95).

SIRT2 deacetylates FOXO3 and increases the DNA-binding activity of FOXO3 resulting in upregulation of FOXO3 target genes, including p27 ${ }^{\mathrm{kip} 1}$, MnSOD and BCL2 like 11 (Bim) (96). In 3T3-L1 adipocytes, FOXO1 is deacetylated by SIRT1, which increases the DNA-binding of FOXO1 to the promoter of peroxisome proliferator activated receptor (PPAR) $\gamma$, and subsequently, suppresses PPAR $\gamma$ expression (97). Inhibition of SIRT2-mediated deacetylation of FOXO1 can increase the retention of FOXO1 in the cytoplasm (98). Furthermore, SIRT3 is the major deacetylase in the mitochondrion (99). SIRT3 deacetylates FOXO3a, which strengthens the DNA-binding of FOXO3a, but does not change the mitochondrial location of FOXO3a (100). It has been demonstrated that FOXO3a forms a complex with SIRT3 and mitochondrial RNA polymerase, binding to mitochondrial DNA, and further increasing the expression of oxidative phosphorylation-associated proteins, ultimately, increasing mitochondrial respiration (59). It has been reported that SIRT6 is pivotal for regulating DNA repair and telomerase (101). In mouse embryonic fibroblasts, SIRT6 inhibition can increase the acetylation level of FOXO3a. However in breast cancer cells, overexpression of SIRT6, which reduces the acetylation level of FOXO3a, weakens the transcription activity of FOXO3a and generates resistance to epirubicin and paclitaxel, which suggests that inhibition of SIRT6 to upregulate the activity of FOXO3a may be a promising therapeutic strategy for breast cancer (78).
Acetylation. Calcium response element-binding binding protein (CBP)/p300 acetylates FOXO1, FOXO3 and FOXO4 (81,102-104). Acetylated FOXO proteins have lower DNA-binding activity compared with normal levels (102). For example, FOXO4 combines with CBP following treatment of human embryonic kidney (HEK)-293T cells with peroxide stress, and acetylated FOXO4 exhibits lower transcription activity (105). Acetylated FOXO proteins tend to be located in the cytoplasm, as acetylated FOXO proteins are more sensitive to phosphorylation induced by Akt (106). The phosphorylation of FOXO proteins induced by Akt following insulin treatment is always accompanied by the acetylation of FOXO proteins (107). Increasing evidence suggests that the acetylation of FOXO proteins regulates its transcriptional activity by altering DNA binding, interaction with transcriptional regulatory proteins, and changes to stability and subcellular localization. FOXOs protein acetylation has weakens its transcriptional activity, however, in pancreatic $\beta$ cells, the acetylation of FOXO1 induced by stress increases its stability and prevents the ubiquitin-proteasome pathway degradation (88). In pancreatic cancer cells, capsaicin enhances the expression of CBP/p300, and reduces SIRT1, SIRT2 and SIRT3. Capsaicin promotes acetylation of FOXO1 and increases nuclear entry, eventually enhancing the DNA-binding. Furthermore, acetylation occurs prior to phosphorylation of FOXO proteins (108). However, the acetylation of FOXO1 caused by p300 does not change its stability. Both wild-type p300, and p300 with an acetyltransferase deletion mutation, enhance the half-life of FOXO1 in HEK-293T and H4IIE rat hepatoma cells, which suggests that acetylation itself is not the key factor required for FOXO1 stability (107).

In addition, FOXO1-CoRepressor (FCoR) protein, which has acetyltransferase activity, influences the interaction between FOXO1 and SIRT1 deacetylase to maintain FOXO1 acetylation and inhibit the activity of FOXO1 in the rat adipocyte (109). It has been demonstrated that the interaction of FOXO1 and SIRT1 is induced by FHL2, which enhances the deacetylation of FOXO1 (94). Currently, FCoR is the only protein known to inhibit the interaction between FOXO1 and SIRT1.

\section{Ubiquitination of FOXO proteins}

Polyubiquitination. The degradation of FOXO proteins depends on the ubiquitin-proteasome pathway. E3 ubiquitin ligase is the key factor involved in FOXO protein ubiquitination and Skp2 is a subunit of the Skp1/cullin 1/F-box protein ubiquitination complex. FOXO1 combines with Skp2 when phosphorylated at the Ser256 loci. Skp2 is the predominant ubiquitin ligase for FOXO1, and induces its polyubiquitination and degradation (18). C-terminus of Hsc70-interacting protein (CHIP) is highly expressed in the heart and blood vessels, and the C-terminus has a cochaperone/ubiquitin ligase with a dual function. Under the stimulus of TNF- $\alpha, \mathrm{CHIP}$ promotes the smooth muscle cells to degrade and ubiquitination (110). Ring finger and WD repeat domain 2 (COP1), a ring-finger E3 ubiquitin ligase regulated by insulin, has a key role in survival of mammalian cells. In hepatocellular carcinoma cells, COP1 degrades FOXO1 and reduces the expression of FOXO1 target genes, including glucose-6-phosphatase and phosphoenolpyruvate carboxykinase (111). 


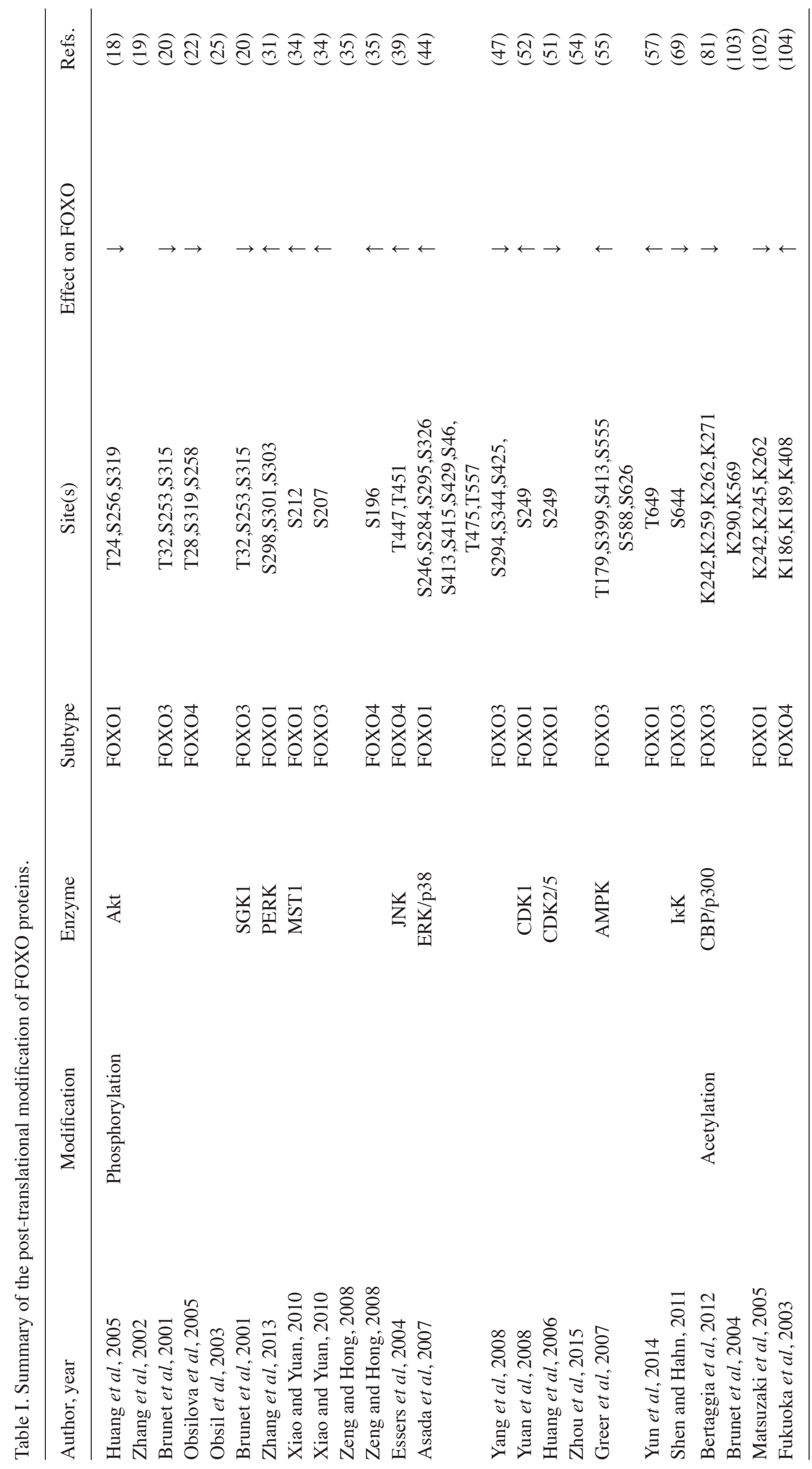




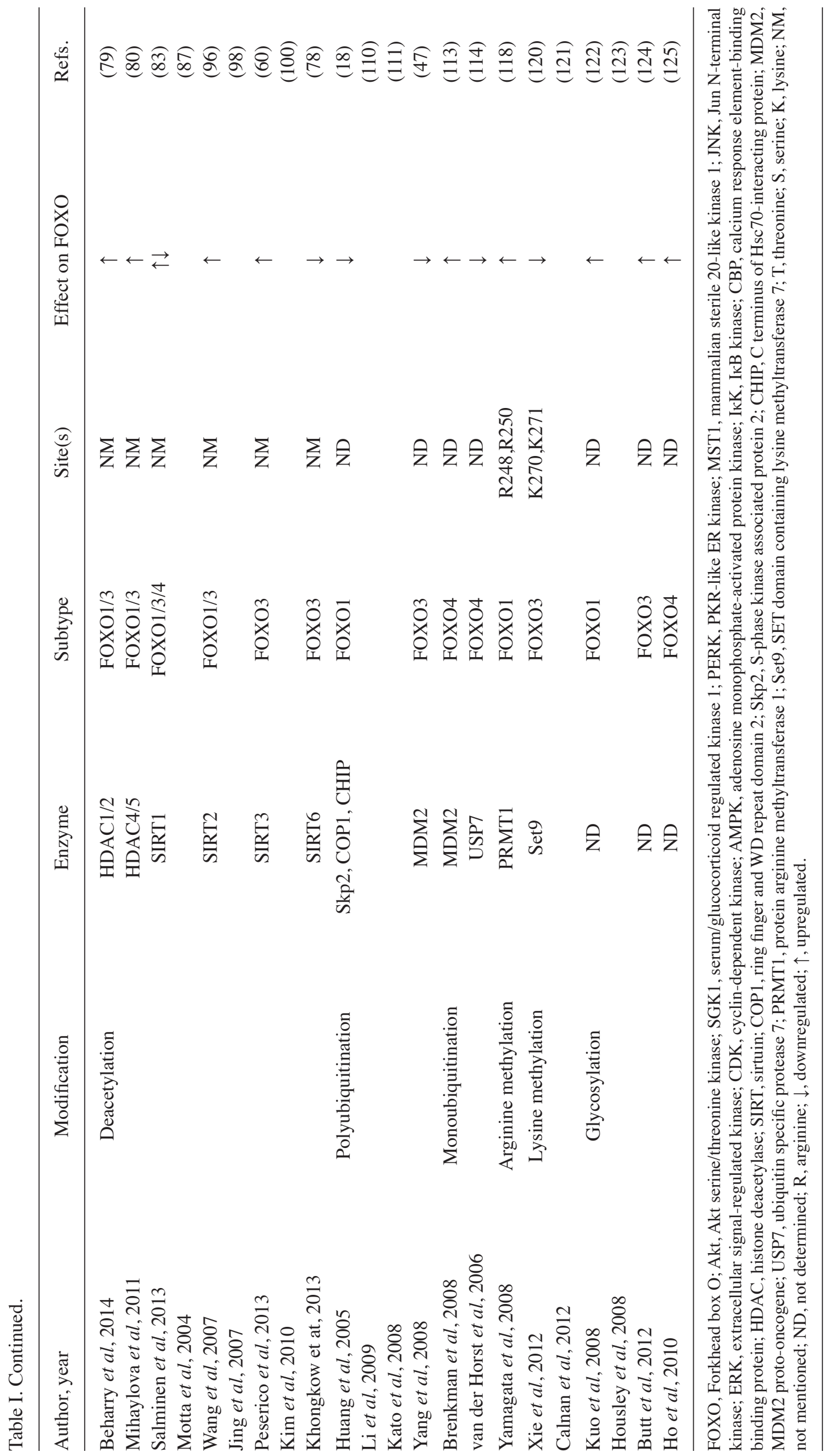


In addition, other kinases may promote FOXO protein degradation though phosphorylating different FOXO loci. ERK regulates the phosphorylation of FOXO3 at Ser294, Ser344 and Ser425 sites that mediate the binding of FOXO3 and MDM2, which results in the polyubiquitination and degradation of FOXO3 (47). Additionally, Ser644 phosphorylation of FOXO3 by I $\mathrm{FB}$ facilities ubiquitination. and ultimately, induces degradation of FOXO3 (112). In conclusion, the phosphorylation of FOXO proteins induced by Akt and I $\mathrm{B}$ is important for mediating polyubiquitination. In addition, FOXO1 acetylation increases the stability following oxidative stress by preventing the polyubiquitination of FOXO1 (88), this result suggests that the activity of FOXO proteins are regulated by various acetylation and ubiquitination events.

Mono-ubiquitination. FOXO proteins activities are also regulated by mono-ubiquitination. However, different from the degradation of FOXO proteins caused by polyubiquitination, mono-ubiquitination increases the activity of FOXO proteins. Typically, MDM2 is considered to be an E3 ubiquitin ligase and can promote the polyubiquitination of FOXO1 and FOXO3. However, in response to oxidative stress, MDM2 induces mono-ubiquitination for $\mathrm{FOXO} 4$, thus, increasing the FOXO4 nuclear relocalization and transcriptional activity (113). However, this effect can be reversed by Herpes-virus-associated ubiquitin specific protease (also termed USP7). USP7 enhances the exclusion of FOXO4 from the nucleus, but does not affect the half-life of FOXO proteins. Furthermore, under oxidative stress conditions, peptidylprolyl cis/trans isomerase, NIMA-interacting 1 (Pin1) binds to FOXO4, and attenuates FOXO4 monoubiquitination and transcriptional activity. Pin1 exerts its effects by preventing nuclear FOXO4 accumulation via stimulation of the activity of the deubiquitinating enzyme HAUSP/USP7, which interacts with and deubiquitinates FOXO4 in response to oxidative stress (114). Additionally, a study reported that USP7 mediates the mono-deubiquitination of FOXO1 in liver cells. USP7-mediated mono-deubiquitination of FOXO1 results in suppression of FOXO1 transcriptional activity through decreased FOXO1 occupancy on the promoters of gluconeogenic genes (115).

In myocardial cells, atrogin-1 (also termed F-box protein 32) inhibits the phosphorylation of FOXO1/3 induced by insulin or IGF-1 and promotes its nuclear entry to activate the FOXO transcriptional activity. For canonical ubiquitin-proteasome degradation, target ubiquitin chains are linked by Lys48 and target proteins for the proteasomal degradation. By contrast, it has been reported that the deposition of Lys63-linked ubiquitin chains on FOXO proteins cannot initiate proteasome degradation. Atrogin-1 modifies the ubiquitination of FOXO proteins in the unconventional manner of Lys63-linked ubiquitin chains. As a consequence, this mechanism enhances FOXO protein transcriptional activity via ubiquitination rather than initiating the ubiquitin-proteasome degradation. Notably, atrogin-1 is also a target gene of FOXO proteins. FOXO proteins with unconventional ubiquitination increase the expression of atrogin-1, which results in a positive feedback loop (116). Furthermore, it has been reported that HDAC6 is not a traditional deacetylation enzyme, and it is directly involved in the protein ubiquitin-proteasome degradation pathway though via its ubiquitin connecting region. HDAC6 is able to interact with atrogin-1 and regulate FOXO protein ubiquitination (117). This finding further demonstrates that FOXO proteins are regulated by acetylation and ubiquitination.

\section{Methylation of FOXO proteins}

Protein arginine methyltransferase (PRMT1) methylates FOXO1 at Arg248 and Arg250. This modification inhibits the phosphorylation of FOXO1 at Ser253 induced by Akt, blocking the exclusion of FOXO1 from nucleus, and consequently, increases apoptosis in response to oxidative stress. However, knock-out of PRMT1 promotes translocation of FOXO proteins from the nucleus to the cytoplasm, thus, accelerating the ubiquitin-proteasome degradation pathway by inducing poly-ubiquitination (118). Methylated FOXO1 remains in the nucleus, where it activates the expression of downstream targets genes. Lysine methylation, induced by other methyltransferases, is important for regulating histones and other proteins (119). Certain studies demonstrated that the methyltransferase, SET domain containing lysine methyltransferase 7 (Set9), methylates FOXO3 at Lys270 and Lys271. Methylated FOXO3 has lower DNA-binding activity and transactivation, and this effect is independent of phosphorylation induced by Akt $(120,121)$. Set9 mediates lysine methylation to suppress the expression of Bim and apoptosis of nerve cells by inducing FOXO3 in response to oxidative stress. Set9 affects FOXO3 specifically, whereas other FOXO protein subtypes are not methylated by Set9 (121).

\section{Glycosylation of FOXO proteins}

Glycosylation is a highly complex process that attaches glycans to protein, lipids or other organic molecules. Proteins are glycosylated by two different types of glycosylation; $\mathrm{N}$ - and $\mathrm{O}$-glycosylation. FOXO1 is a substrate for O-glycosylation, which induces upregulation of glucose-6-phosphatase (122) and other gluconeogenic genes expression (123), however it does not influence the nuclear-cytoplasm shuttling of FOXO proteins. This finding suggest that glycosylation is a primary PTM for regulating FOXO1 activity. Additionally, it has been reported that partial Ser/Thr sites of FOXO3 may be targets for O-glycosylation (124). Furthermore, in response to oxidative stress, O-linked $\mathrm{N}$-acetylglucosamine transferase glycosylates FOXO4, which enhances the transcriptional activity of FOXO4 (125).

\section{Conclusion}

The present review describes the recent findings regarding PTMs of FOXO proteins, and an increasing number of reports indicate that different stimuli activate different FOXO proteins PTMs, and FOXO transcriptional activity is not regulated by a single PTMs. Furthermore, with the same stimulus, different PTMs may exert opposing effects to ultimately maintain FOXO protein activity at a particular level. Certain enzymes that modify the FOXO proteins are also downstream target genes of the FOXO transcription factors. Thus, these enzymes create a positive feedback loop to regulate the FOXO proteins. Occasionally, different modifications affect each other via association with certain 
factors, therefore, influencing the activity of FOXO proteins directly and indirectly. In conclusion, FOXO protein PTMs have a complex topological relationship and further investigation of the association between disease and FOXO PTMs is required.

\section{References}

1. Weigel D, Jürgens G, Küttner F, Seifert E and Jäckle H: The homeotic gene fork head encodes a nuclear protein and is expressed in the terminal regions of the Drosophila embryo. Cell 57: 645-658, 1989.

2. Weigel D and Jäckle H: The fork head domain: A novel DNA binding motif of eukaryotic transcription factors? Cell 63: 455-456, 1990.

3. Kaufmann E and Knöchel W: Five years on the wings of fork head. Mech Dev 57: 3-20, 1996.

4. Furuyama T, Nakazawa T, Nakano I and Mori N: Identification of the differential distribution patterns of mRNAs and consensus binding sequences for mouse DAF-16 homologues. Biochem J 349: 629-634, 2000.

5. Kaestner KH, Knochel W and Martinez DE: Unified nomenclature for the winged helix/forkhead transcription factors. Genes Dev 14: 142-146, 2000.

6. Myatt SS and Lam EW: The emerging roles of forkhead box (Fox) proteins in cancer. Nat Rev Cancer 7: 847-859, 2007.

7. Shen X, Cui J and Gong Q: Fox gene loci in Takifugu rubripes and Tetraodon nigroviridis genomes and comparison with those of medaka and zebrafish genomes. Genome 54: 965-972, 2011.

8. Greer EL and Brunet A: FOXO transcription factors at the interface between longevity and tumor suppression. Oncogene 24: 7410-7425, 2005

9. Jacobs FM, van der Heide LP, Wijchers PJ, Burbach JP, Hoekman MF and Smidt MP: FoxO6, a novel member of the FoxO class of transcription factors with distinct shuttling dynamics. J Biol Chem 278: 35959-35967, 2003.

10. Hu HJ, Zhang LG, Wang ZH and Guo XX: FoxO6 inhibits cell proliferation in lung carcinoma through up-regulation of USP7. Mol Med Rep 12: 575-580, 2015.

11. Kim DH, Zhang T, Lee S, Calabuig-Navarro V, Yamauchi J, Piccirillo A, Fan Y, Uppala R, Goetzman E and Dong HH: FoxO6 integrates insulin signaling with MTP for regulating VLDL production in the liver. Endocrinology 155: 1255-1267, 2014.

12. Larroux C, Luke GN, Koopman P, Rokhsar DS, Shimeld SM and Degnan BM: Genesis and expansion of metazoan transcription factor gene classes. Mol Biol Evol 25: 980-996, 2008.

13. Lapierre LR, Kumsta C, Sandri M, Ballabio A and Hansen M Transcriptional and epigenetic regulation of autophagy in aging. Autophagy 11: 867-880, 2015.

14. Edmonds JW, Prasain JK, Dorand D, Yang Y, Hoang HD, Vibbert J, Kubagawa HM and Miller MA: Insulin/FOXO signaling regulates ovarian prostaglandins critical for reproduction. Dev Cell 19: 858-871, 2010.

15. Lin L, Hron JD and Peng SL: Regulation of NF-kappaB, Th activation, and autoinflammation by the forkhead transcription factor Foxo3a. Immunity 21: 203-213, 2004.

16. Smith WW, Norton DD, Gorospe M, Jiang H, Nemoto S Holbrook NJ, Finkel T and Kusiak JW: Phosphorylation of p66Shc and forkhead proteins mediates Abeta toxicity. J Cell Biol 169: 331-339, 2005.

17. van der Vos KE and Coffer PJ: The extending network of FOXO transcriptional target genes. Antioxid Redox Signal 14: 579-592, 2011.

18. Huang H, Regan KM, Wang F, Wang D, Smith DI, van Deursen JM and Tindall DJ: Skp2 inhibits FOXO1 in tumor suppression through ubiquitin-mediated degradation. Proc Natl Acad Sci USA 102: 1649-1654, 2005.

19. Zhang X, Gan L, Pan H, Guo S, He X, Olson ST, Mesecar A Adam S and Unterman TG: Phosphorylation of serine 256 suppresses transactivation by FKHR (FOXO1) by multiple mechanisms. Direct and indirect effects on nuclear/cytoplasmic shuttling and DNA binding. J Biol Chem 277: 45276-45284, 2002.

20. Brunet A, Park J, Tran H, Hu LS, Hemmings BA and Greenberg ME: Protein kinase SGK mediates survival signals by phosphorylating the forkhead transcription factor FKHRL1 (FOXO3a). Mol Cell Biol 21: 952-965, 2001.
21. Brunet A, Kanai F, Stehn J, Xu J, Sarbassova D, Frangioni JV, Dalal SN, DeCaprio JA, Greenberg ME and Yaffe MB: 14-3-3 transits to the nucleus and participates in dynamic nucleocytoplasmic transport. J Cell Biol 156: 817-828, 2002.

22. Obsilova V, Vecer J, Herman P, Pabianova A, Sulc M, Teisinger J, Boura E and Obsil T: 14-3-3 Protein interacts with nuclear localization sequence of forkhead transcription factor FoxO4. Biochemistry 44: 11608-11617, 2005.

23. Rena G, Prescott AR, Guo S, Cohen P and Unterman TG: Roles of the forkhead in rhabdomyosarcoma (FKHR) phosphorylation sites in regulating 14-3-3 binding, transactivation and nuclear targetting. Biochem J 354: 605-612, 2001.

24. Tsai WC, Bhattacharyya N, Han LY, Hanover JA and Rechler MM: Insulin inhibition of transcription stimulated by the forkhead protein Foxol is not solely due to nuclear exclusion. Endocrinology 144: 5615-5622, 2003

25. Obsil T, Ghirlando R, Anderson DE, Hickman AB and Dyda F: Two 14-3-3 binding motifs are required for stable association of Forkhead transcription factor FOXO4 with 14-3-3 proteins and inhibition of DNA binding. Biochemistry 42: 15264-15272, 2003

26. Plas DR and Thompson CB: Akt activation promotes degradation of tuberin and FOXO3a via the proteasome. J Biol Chem 278: 12361-12366, 2003.

27. Aoki M, Jiang $\mathrm{H}$ and Vogt PK: Proteasomal degradation of the FoxO1 transcriptional regulator in cells transformed by the P3k and Akt oncoproteins. Proc Natl Acad Sci USA 101: 13613-13617, 2004.

28. Matsuzaki H, Daitoku H, Hatta M, Tanaka K and Fukamizu A: Insulin-induced phosphorylation of FKHR (Foxo1) targets to proteasomal degradation. Proc Natl Acad Sci USA 100: 11285-11290, 2003.

29. Santo EE, Stroeken P, Sluis PV, Koster J, Versteeg R and Westerhout EM: FOXO3a is a major target of inactivation by $\mathrm{PI} 3 \mathrm{~K} / \mathrm{AKT}$ signaling in aggressive neuroblastoma. Cancer Res 73: 2189-2198, 2013.

30. Alon U: Network motifs: Theory and experimental approaches. Nat Rev Genet 8: 450-461, 2007.

31. Zhang W, Hietakangas V, Wee S, Lim SC, Gunaratne J and Cohen SM: ER stress potentiates insulin resistance through PERK-mediated FOXO phosphorylation. Genes Dev 27: 441-449, 2013.

32. Mounir Z, Krishnamoorthy JL, Wang S, Papadopoulou B, Campbell S, Muller WJ, Hatzoglou M and Koromilas AE: Akt determines cell fate through inhibition of the PERK-eIF2 $\alpha$ phosphorylation pathway. Sci Signal 4: ra62, 2011.

33. Bobrovnikova-Marjon E, Pytel D, Riese MJ, Vaites LP, Singh N, Koretzky GA, Witze ES and Diehl JA: PERK utilizes intrinsic lipid kinase activity to generate phosphatidic acid, mediate Akt activation, and promote adipocyte differentiation. Mol Cell Biol 32: 2268-2278, 2012.

34. Xiao L and Yuan Z: Redemystifying MST1/hippo signaling. Protein Cell 1: 706-708, 2010.

35. Zeng Q and Hong W: The emerging role of the hippo pathway in cell contact inhibition, organ size control and cancer development in mammals. Cancer Cell 13: 188-192, 2008.

36. Lehtinen MK, Yuan Z, Boag PR, Yang Y, Villén J, Becker EB, DiBacco S, de la Iglesia N, Gygi S, Blackwell TK and Bonni A: A conserved MST-FOXO signaling pathway mediates oxidative-stress responses and extends life span. Cell 125: 987-1001, 2006.

37. Yuan Z, Lehtinen MK, Merlo P, Villén J, Gygi S and Bonni A: Regulation of neuronal cell death by MST1-FOXO1 signaling. J Biol Chem 284: 11285-11292, 2009.

38. Valis K, Prochazka L, Boura E, Chladova J, Obsil T, Rohlena J, Truksa J, Dong LF, Ralph SJ and Neuzil J: Hippo/Mst1 stimulates transcription of the proapoptotic mediator NOXA in a FoxO1-dependent manner. Cancer Res 71: 946-954, 2011.

39. Essers MA, Weijzen S, de Vries-Smits AM, Saarloos I, de Ruiter ND, Bos JL and Burgering BM: FOXO transcription factor activation by oxidative stress mediated by the small GTPase Ral and JNK. EMBO J 23: 4802-4812, 2004.

40. van den Berg MC, van Gogh IJ, Smits AM, van Triest M, Dansen TB, Visscher M, Polderman PE, Vliem MJ, Rehmann H and Burgering BM: The small GTPase RALA controls c-Jun $\mathrm{N}$-terminal kinase-mediated FOXO activation by regulation of a JIP1 scaffold complex. J Biol Chem 288: 21729-21741, 2013.

41. Sunayama J, Tsuruta F, Masuyama N and Gotoh Y: JNK antagonizes Akt-mediated survival signals by phosphorylating 14-3-3. J Cell Biol 170: 295-304, 2005. 
42. Kawamori D, Kaneto H, Nakatani Y, Matsuoka TA, Matsuhisa M, Hori M and Yamasaki Y: The forkhead transcription factor Foxo1 bridges the JNK pathway and the transcription factor PDX-1 through its intracellular translocation. J Biol Chem 281: 1091-1098, 2006.

43. Song JJ and Lee YJ: Cross-talk between JIP3 and JIP1 during glucose deprivation: SEK1-JNK2 and Akt1 act as mediators. J Biol Chem 280: 26845-26855, 2005.

44. Asada S, Daitoku H, Matsuzaki H, Saito T, Sudo T, Mukai H, Iwashita S, Kako K, Kishi T, Kasuya Y and Fukamizu A Mitogen-activated protein kinases, Erk and p38, phosphorylate and regulate Foxo1. Cell Signal 19: 519-527, 2007.

45. Ho KK, McGuire VA, Koo CY, Muir KW, de Olano N, Maifoshie E, Kelly DJ, McGovern UB, Monteiro LJ, Gomes AR, et al: Phosphorylation of FOXO3a on Ser-7 by p38 promotes its nuclear localization in response to doxorubicin. J Biol Chem 287: 1545-1555, 2012.

46. Lin A, Yao J, Zhuang L, Wang D, Han J, Lam EW and Gan B: The FoxO-BNIP3 axis exerts a unique regulation of mTORC1 and cell survival under energy stress. Oncogene 33: 3183-3194, 2014.

47. Yang JY, Zong CS, Xia W, Yamaguchi H, Ding Q, Xie X, Lang JY, Lai CC, Chang CJ, Huang WC, et al: ERK promotes tumorigenesis by inhibiting FOXO3a via MDM2-mediated degradation. Nat Cell Biol 10: 138-148, 2008

48. Hu Y, Wang X, Zeng L, Cai DY, Sabapathy K, Goff SP, Firpo EJ and Li B: ERK phosphorylates p66shcA on Ser36 and subsequently regulates p27kip1 expression via the Akt-FOXO3a pathway: Implication of p27kip1 in cell response to oxidative stress. Mol Biol Cell 16: 3705-3718, 2005.

49. Pramod S and Shivakumar K: Mechanisms in cardiac fibroblast growth: An obligate role for Skp2 and FOXO3a in ERK1/2 MAPK-dependent regulation of p27kip1. Am J Physiol Heart Circ Physiol 306: H844-H855, 2014.

50. Kodiha M, Banski P and Stochaj U: Interplay between MEK and PI3Kinase signaling regulates the subcellular localization of protein kinases ERK1/2 and Akt upon oxidative stress. FEBS Lett 583: 1987-1993, 2009.

51. Huang H, Regan KM, Lou Z, Chen J and Tindall DJ: CDK2-dependent phosphorylation of FOXO1 as an apoptotic response to DNA damage. Science 314: 294-297, 2006.

52. Yuan Z, Becker EB, Merlo P, Yamada T, DiBacco S, Konishi Y, Schaefer EM and Bonni A: Activation of FOXO1 by Cdk1 in cycling cells and postmitotic neurons. Science 319: 1665-1668, 2008.

53. Liu P, Kao TP and Huang H: CDK1 promotes cell proliferation and survival via phosphorylation and inhibition of FOXO1 transcription factor. Oncogene 27: 4733-4744, 2008.

54. Zhou J, Li H, Li X, Zhang G, Niu Y, Yuan Z, Herrup K, Zhang $\mathrm{YW}, \mathrm{Bu} \mathrm{G}, \mathrm{Xu} \mathrm{H}$ and Zhang $\mathrm{J}$ : The roles of Cdk5-mediated subcellular localization of FOXO1 in neuronal death. J Neurosci 35: 2624-2635, 2015.

55. Greer EL, Oskoui PR, Banko MR, Maniar JM, Gygi MP, Gygi SP and Brunet A: The energy sensor AMP-activated protein kinase directly regulates the mammalian FOXO3 transcription factor. J Biol Chem 282: 30107-30119, 2007

56. Chiacchiera F, Matrone A, Ferrari E, Ingravallo G, Lo Sasso G, Murzilli S, Petruzzelli M, Salvatore L, Moschetta A and Simone C: p38alpha blockade inhibits colorectal cancer growth in vivo by inducing a switch from HIF1alpha- to FoxO-dependent transcription. Cell Death Differ 16 1203-1214, 2009.

57. Yun H, Park S, Kim MJ, Yang WK, Im DU, Yang KR, Hong J, Choe W, Kang I, Kim SS and Ha J: AMP-activated protein kinase mediates the antioxidant effects of resveratrol through regulation of the transcription factor FoxO1. FEBS J 281: 4421-4438, 2014

58. Chiacchiera F and Simone C: The AMPK-FoxO3A axis as a target for cancer treatment. Cell Cycle 9: 1091-1096, 2010.

59. Nakashima K and Yakabe Y: AMPK activation stimulates myofibrillar protein degradation and expression of atrophy-related ubiquitin ligases by increasing FOXO transcription factors in $\mathrm{C} 2 \mathrm{C} 12$ myotubes. Biosci Biotechnol Biochem 71: 1650-1656, 2007.

60. Peserico A, Chiacchiera F, Grossi V, Matrone A Latorre D, Simonatto M, Fusella A, Ryall JG, Finley LW, Haigis MC, et al: A novel AMPK-dependent FoxO3A-SIRT3 intramitochondrial complex sensing glucose levels. Cell Mol Life Sci 70: 2015-2029, 2013.
61. Li XN, Song J, Zhang L, LeMaire SA, Hou X, Zhang C, Coselli JS, Chen L, Wang XL, Zhang Y and Shen YH: Activation of the AMPK-FOXO3 pathway reduces fatty acid-induced increase in intracellular reactive oxygen species by upregulating thioredoxin. Diabetes 58: 2246-2257, 2009.

62. Sengupta A, Molkentin JD, Paik JH, DePinho RA and Yutzey KE: FoxO transcription factors promote cardiomyocyte survival upon induction of oxidative stress. J Biol Chem 286 : 7468-7478, 2011.

63. Greer EL, Dowlatshahi D, Banko MR, Villen J, Hoang K, Blanchard D, Gygi SP and Brunet A: An AMPK-FOXO pathway mediates longevity induced by a novel method of dietary restriction in C. elegans. Curr Biol 17: 1646-1656, 2007.

64. Tullet JM, Araiz C, Sanders MJ, Au C, Benedetto A, Papatheodorou I, Clark E, Schmeisser K, Jones D, Schuster EF, et al: DAF-16/FoxO directly regulates an atypical AMP-activated protein kinase gamma isoform to mediate the effects of insulin/IGF-1 signaling on aging in Caenorhabditis elegans. PLoS Genet 10: e1004109, 2014.

65. Eijkelenboom A, Mokry M, de Wit E, Smits LM, Polderman PE, van Triest MH, van Boxtel R, Schulze A, de Laat W, Cuppen E and Burgering BM: Genome-wide analysis of $\mathrm{FOXO} 3$ mediated transcription regulation through RNA polymerase II profiling. Mol Syst Biol 9: 638, 2013.

66. Lützner N, De-Castro Arce J and Rösl F: Gene expression of the tumour suppressor LKB1 is mediated by Sp1, NF-Y and FOXO transcription factors. PLoS One 7: e32590, 2012.

67. Cantó C, Gerhart-Hines Z, Feige JN, Lagouge M, Noriega L, Milne JC, Elliott PJ, Puigserver P and Auwerx J: AMPK regulates energy expenditure by modulating NAD+ metabolism and SIRT1 activity. Nature 458: 1056-1060, 2009.

68. Tezil T, Bodur C, Kutuk O and Basaga H: IKK- $\beta$ mediates chemoresistance by sequestering FOXO3; a critical factor for cell survival and death. Cell Signal 24: 1361-1368, 2012.

69. Shen RR and Hahn WC: Emerging roles for the non-canonical IKKs in cancer. Oncogene 30: 631-641, 2011.

70. Guo JP, Tian W, Shu S, Xin Y, Shou C and Cheng JQ: IKBKE phosphorylation and inhibition of FOXO3a: A mechanism of IKBKE oncogenic function. PLoS One 8: e63636, 2013

71. Guo JP, Coppola D and Cheng JQ: IKBKE protein activates Akt independent of phosphatidylinositol 3-kinase/PDK1/mTORC2 and the pleckstrin homology domain to sustain malignant transformation. J Biol Chem 286: 37389-37398, 2011.

72. Luron L, Saliba D, Blazek K, Lanfrancotti A and Udalova IA FOXO3 as a new IKK- $\varepsilon$-controlled check-point of regulation of IFN- $\beta$ expression. Eur J Immunol 42: 1030-1037, 2012.

73. Chapuis N, Park S, Leotoing L, Tamburini J, Verdier F, Bardet V, Green AS, Willems L, Agou F, Ifrah N, et al: IkB kinase overcomes PI3K/Akt and ERK/MAPK to control FOXO3a activity in acute myeloid leukemia. Blood 116: 4240-4250, 2010.

74. Wilson MK, McWhirter SM, Amin RH, Huang D and Schlissel MS: Abelson virus transformation prevents TRAIL expression by inhibiting FoxO3a and NF-kappaB. Mol Cells 29: 333-341, 2010.

75. Li Z, Zhang H, Chen Y, Fan L and Fang J: Forkhead transcription factor FOXO3a protein activates nuclear factor $\mathrm{kB}$ through B-cell lymphoma/leukemia 10 (BCL10) protein and promotes tumor cell survival in serum deprivation. J Biol Chem 287: 17737-17745, 2012.

76. West AC and Johnstone RW: New and emerging HDAC inhibitors for cancer treatment. J Clin Invest 124: 30-39, 2014.

77. Auburger G, Gispert S and Jendrach M: Mitochondrial acetylation and genetic models of Parkinson's disease. Prog Mol Biol Transl Sci 127: 155-182, 2014

78. Khongkow M, Olmos Y, Gong C, Gomes AR, Monteiro LJ, Yagüe E, Cavaco TB, Khongkow P, Man EP, Laohasinnarong S, et al: SIRT6 modulates paclitaxel and epirubicin resistance and survival in breast cancer. Carcinogenesis 34: 1476-1486, 2013.

79. Beharry AW, Sandesara PB, Roberts BM, Ferreira LF, Senf SM and Judge AR: HDACl activates FoxO and is both sufficient and required for skeletal muscle atrophy. J Cell Sci 127: 1441-1453, 2014.

80. Mihaylova MM, Vasquez DS, Ravnskjaer K, Denechaud PD, Yu RT, Alvarez JG, Downes M, Evans RM, Montminy M and Shaw RJ: Class IIa histone deacetylases are hormone-activated regulators of FOXO and mammalian glucose homeostasis. Cell 145: 607-621, 2011 
81. Bertaggia E, Coletto L and Sandri M: Posttranslational modifications control FoxO3 activity during denervation. Am J Physiol Cell Physiol 302: C587-C596, 2012.

82. Senf SM, Sandesara PB, Reed SA and Judge AR: p300 Acetyltransferase activity differentially regulates the localization and activity of the FOXO homologues in skeletal muscle. Am J Physiol Cell Physiol 300: C1490-C1501, 2011.

83. Salminen A, Kaarniranta K and Kauppinen A: Crosstalk between oxidative stress and SIRT1: Impact on the aging process. Int J Mol Sci 14: 3834-3859, 2013.

84. Xiong S, Salazar G, Patrushev N and Alexander RW: FoxO1 mediates an autofeedback loop regulating SIRT1 expression. J Biol Chem 286: 5289-5299, 2011.

85. Wang YQ, Cao Q, Wang F, Huang LY, Sang TT, Liu F and Chen SY: SIRT1 protects against oxidative stress-induced endothelia progenitor cells apoptosis by inhibiting FOXO3a via FOXO3a ubiquitination and degradation. J Cell Physiol 230: 2098-2107, 2015.

86. Wang F, Chan CH, Chen K, Guan X, Lin HK and Tong Q: Deacetylation of FOXO3 by SIRT1 or SIRT2 leads to Skp2-mediated FOXO3 ubiquitination and degradation. Oncogene 31: 1546-1557, 2012.

87. Motta MC, Divecha N, Lemieux M, Kamel C, Chen D, Gu W, Bultsma Y, McBurney M and Guarente L: Mammalian SIRT1 represses forkhead transcription factors. Cell 116: 551-563, 2004

88. Kitamura YI, Kitamura T, Kruse JP, Raum JC, Stein R, Gu W and Accili D: FoxO1 protects against pancreatic beta cell failure through NeuroD and MafA induction. Cell Metab 2: 153-163, 2005.

89. Aquilano K, Baldelli S, Pagliei B and Ciriolo MR: Extranuclear localization of SIRT1 and PGC-1 $\alpha$ : An insight into possible roles in diseases associated with mitochondrial dysfunction. Curr Mol Med 13: 140-154, 2013.

90. Morselli E, Mariño G, Bennetzen MV, Eisenberg T, Megalou E, Schroeder S, Cabrera S, Bénit P, Rustin P, Criollo A, et al: Spermidine and resveratrol induce autophagy by distinct pathways converging on the acetylproteome. J Cell Biol 192: 615-629, 2011.

91. Ng F and Tang BL: Sirtuins' modulation of autophagy. J Cell Physiol 228: 2262-2270, 2013.

92. Yun JM, Chien A, Jialal I and Devaraj S: Resveratrol up-regulates SIRT1 and inhibits cellular oxidative stress in the diabetic milieu: Mechanistic insights. J Nutr Biochem 23: 699-705, 2012.

93. Sin TK, Yung BY and Siu PM: Modulation of SIRT1-Foxo1 signaling axis by resveratrol: Implications in skeletal muscle aging and insulin resistance. Cell Physiol Biochem 35: 541-552, 2015.

94. Yang Y, Hou H, Haller EM, Nicosia SV and Bai W: Suppression of FOXO1 activity by FHL2 through SIRT1-mediated deacetylation. EMBO J 24: 1021-1032, 2005.

95. Liu X, Greer C and Secombe J: KDM5 interacts with Foxo to modulate cellular levels of oxidative stress. PLoS Genet 10 : e1004676, 2014

96. Wang F, Nguyen M, Qin FX and Tong Q: SIRT2 deacetylates FOXO3a in response to oxidative stress and caloric restriction. Aging Cell 6: 505-514, 2007.

97. Wang F and Tong Q: SIRT2 suppresses adipocyte differentiation by deacetylating FOXO1 and enhancing FOXO1's repressive interaction with PPARgamma. Mol Biol Cell 20: 801-808, 2009.

98. Jing E, Gesta S and Kahn CR: SIRT2 regulates adipocyte differentiation through FoxO1 acetylation/deacetylation. Cell Metab 6 105-114, 2007

99. Lombard DB, Alt FW, Cheng HL, Bunkenborg J, Streeper RS, Mostoslavsky R, Kim J, Yancopoulos G, Valenzuela D, Murphy A, et al: Mammalian Sir2 homolog SIRT3 regulates global mitochondrial lysine acetylation. Mol Cell Biol 27: 8807-8814, 2007.

100. Kim HS, Patel K, Muldoon-Jacobs K, Bisht KS, Aykin-Burns N, Pennington JD, van der Meer R, Nguyen P, Savage J, Owens KM, et al: SIRT3 is a mitochondria-localized tumor suppressor required for maintenance of mitochondrial integrity and metabolism during stress. Cancer Cell 17: 41-52, 2010.

101. Jia G, Su L, Singhal S and Liu X: Emerging roles of SIRT6 on telomere maintenance, DNA repair, metabolism and mammalian aging. Mol Cell Biochem 364: 345-350, 2012.

102. Matsuzaki H, Daitoku H, Hatta M, Aoyama H, Yoshimochi K and Fukamizu A: Acetylation of Foxol alters its DNA-binding ability and sensitivity to phosphorylation. Proc Natl Acad Sci USA 102: 11278-11283, 2005.

103. Brunet A, Sweeney LB, Sturgill JF, Chua KF, Greer PL, Lin Y, Tran H, Ross SE, Mostoslavsky R, Cohen HY, et al: Stress-dependent regulation of FOXO transcription factors by the SIRT1 deacetylase. Science 303: 2011-2015, 2004.
104. Fukuoka M, Daitoku H, Hatta M, Matsuzaki H, Umemura S and Fukamizu A: Negative regulation of forkhead transcription factor AFX (Foxo4) by CBP-induced acetylation. Int J Mol Med 12: 503-508, 2003

105. van der Horst A, Tertoolen LG, de Vries-Smits LM, Frye RA, Medema RH and Burgering BM: FOXO4 is acetylated upon peroxide stress and deacetylated by the longevity protein hSir2(SIRT1). J Biol Chem 279: 28873-28879, 2004.

106. Qiang L, Banks AS and Accili D: Uncoupling of acetylation from phosphorylation regulates FoxO1 function independent of its subcellular localization. J Biol Chem 285: 27396-27401, 2010.

107. Perrot V and Rechler MM: The coactivator p300 directly acetylates the forkhead transcription factor Foxol and stimulates Foxol-induced transcription. Mol Endocrinol 19: 2283-2298, 2005.

108. Pramanik KC, Fofaria NM, Gupta P and Srivastava SK: CBP-mediated FOXO-1 acetylation inhibits pancreatic tumor growth by targeting SirT. Mol Cancer Ther 13: 687-698, 2014

109. Nakae J, Cao Y, Hakuno F, Takemori H, Kawano Y, Sekioka R, Abe T, Kiyonari H, Tanaka T, Sakai J, et al: Novel repressor regulates insulin sensitivity through interaction with Foxol. EMBO J 31: 2275-2295, 2012

110. Li F, Xie P, Fan Y, Zhang H, Zheng L, Gu D, Patterson C and Li H: $\mathrm{C}$ terminus of Hsc70-interacting protein promotes smooth muscle cell proliferation and survival through ubiquitin-mediated degradation of FoxO1. J Biol Chem 284: 20090-20098, 2009.

111. Kato S, Ding J, Pisck E, Jhala US and Du K: COP1 functions as a FoxO1 ubiquitin E3 ligase to regulate FoxO1-mediated gene expression. J Biol Chem 283: 35464-35473, 2008.

112. Hu MC, Lee DF, Xia W, Golfman LS, Ou-Yang F, Yang JY, Zou Y, Bao S, Hanada N, Saso H, et al: IkappaB kinase promotes tumorigenesis through inhibition of forkhead FOXO3a. Cell 117: 225-237, 2004.

113. Brenkman AB, de Keizer PL, van den Broek NJ, Jochemsen AG and Burgering BM: Mdm2 induces mono-ubiquitination of FOXO4. PLoS One 3: e2819, 2008.

114. van der Horst A, de Vries-Smits AM,Brenkman AB, van Triest MH, van den Broek N, Colland F, Maurice MM and Burgering BM: FOXO4 transcriptional activity is regulated by monoubiquitination and USP7/HAUSP. Nat Cell Biol 8: 1064-1073, 2006.

115. Hall JA, Tabata M, Rodgers JT and Puigserver P: USP7 attenuates hepatic gluconeogenesis through modulation of FoxO1 gene promoter occupancy. Mol Endocrinol 28: 912-924, 2014.

116. Li HH, Willis MS, Lockyer P, Miller N, McDonough H, Glass DJ and Patterson C: Atrogin-1 inhibits Akt-dependent cardiac hypertrophy in mice via ubiquitin-dependent coactivation of Forkhead proteins. J Clin Invest 117: 3211-3223, 2007.

117. Ratti F, Ramond F, Moncollin V, Simonet T, Milan G, Méjat A, Thomas JL, Streichenberger N, Gilquin B, Matthias P, et al: Histone deacetylase 6 is a FoxO transcription factor-dependent effector in skeletal muscle atrophy. J Biol Chem 290: 4215-4224, 2015

118. Yamagata K, Daitoku H, Takahashi Y, Namiki K, Hisatake K, Kako K, Mukai H, Kasuya Y and Fukamizu A: Arginine methylation of FOXO transcription factors inhibits their phosphorylation by Akt. Mol Cell 32: 221-231, 2008.

119. Huang $\mathbf{J}$ and Berger SL: The emerging field of dynamic lysine methylation of non-histone proteins. Curr Opin Genet Dev 18: $152-158,2008$

120. Xie Q, Hao Y, Tao L, Peng S, Rao C, Chen H, You H, Dong MQ and Yuan Z: Lysine methylation of FOXO3 regulates oxidative stress-induced neuronal cell death. EMBO Rep 13: 371-377, 2012.

121. Calnan DR, Webb AE, White JL, Stowe TR, Goswami T, Shi X, Espejo A, Bedford MT, Gozani O, Gygi SP and Brunet A: Methylation by Set 9 modulates FoxO3 stability and transcriptional activity. Aging (Albany NY) 4: 462-479, 2012.

122. Kuo M, Zilberfarb V, Gangneux N, Christeff N and Issad T: $\mathrm{O}$-glycosylation of FoxO1 increases its transcriptional activity towards the glucose 6-phosphatase gene. FEBS Lett 582: 829-834, 2008

123. Housley MP, Rodgers JT, Udeshi ND, Kelly TJ, Shabanowitz J, Hunt DF, Puigserver P and Hart GW: O-GlcNAc regulates FoxO activation in response to glucose. J Biol Chem 283: 16283-16292, 2008.

124. Butt AM, Feng D, Idrees M, Tong Y and Lu J: Computational identification and modeling of crosstalk between phosphorylation, $\mathrm{O}-\beta$-glycosylation and methylation of FoxO3 and implications for cancer therapeutics. Int J Mol Sci 13: 2918-2938, 2012.

125. Ho SR, Wang K, Whisenhunt TR, Huang P, Zhu X, Kudlow JE and Paterson AJ: O-GlcNAcylation enhances FOXO4 transcriptional regulation in response to stress. FEBS Lett 584: 49-54, 2010. 